\title{
NONLINEAR INTERACTION BETWEEN MAGNETIC FIELDS AND SPIRAL ARMS
}

\author{
M. CHIBA* \\ Max-Planck-Institut für Radioastronomie, Auf dem Hügel 69, W-5300 Bonn, Germany
}

\begin{abstract}
The evolution of galactic magnetic fields under the influence of spiral arms is investigated numerically by 2-dimensional simulation. We compute several models, especially when the swing-excitation mechanism works. We consider also the modification of the mean velocity fields driven by the Lorentz force of the growing magnetic fields. The characteristic structure of magnetic fields around the arm in each model is compared with the observations.
\end{abstract}

Key words: Galactic Magnetic Fields - Spiral Arms - Nonlinear Interaction

\section{Introduction}

The spiral arms govern gas dynamics in galaxies, and thus we cannot neglect their effects when we think about galactic magnetic fields. In particular, the result of a simple disk dynamo is readily modified. In order to see their effects on magnetic fields, we have done the 2-dimensional numerical simulation.

\section{Model assumptions}

The calculated area is the local region around the arm in a Cartesian frame $(x, y, z)$ which rotates at the pattern speed $\Omega_{p}$ of the spiral arm. The $x$-axis is perpendicular to the arm, $y$-axis parallel to it, and $z$ toward the halo. The gas flow is a steady, vertically extending shock wave due to a tightly-wound spiral potential (e.g. Tubbs 1980). As a feedback from the magnetic fields, we consider the effects of meanvelocity fields induced by the Lorentz forces. The region above the disk is currentfree, and at the boundaries for $x$ we assume the change of the direction of the magnetic fields (BSS fields).

Here we briefly describe the results for 3 models. The model 1 allows the complete coupling between dynamo and density waves (Swing Excitation), so that the pattern speed of the magnetic fields in the $x$-direction is equal to that of the density wave. The model 2 is the version of simple disk dynamo for the model 2 without density waves. Finally the model 3 corresponds to the non-oscillatory disk dynamo disturbed by density wave.

\section{Results}

In the model 1, because of the occurrence of swing excitation, the magnetic energy grows. Note that this case is otherwise damping without density waves (the model 2 ). When the magnetic energy is comparable to the energy of gaseous disturbance, the magnetic field ceases to.grow, and settles into the equilibrium state. On the other hand, the model 3 should lead to the non-oscillatory growth of the magnetic field in simple dynamo, but in the presence of density waves, it does not grow due to the change of velocity fields.

* Permanent address: Astronomical Institute, Tohoku University, Sendai 980, Japan 

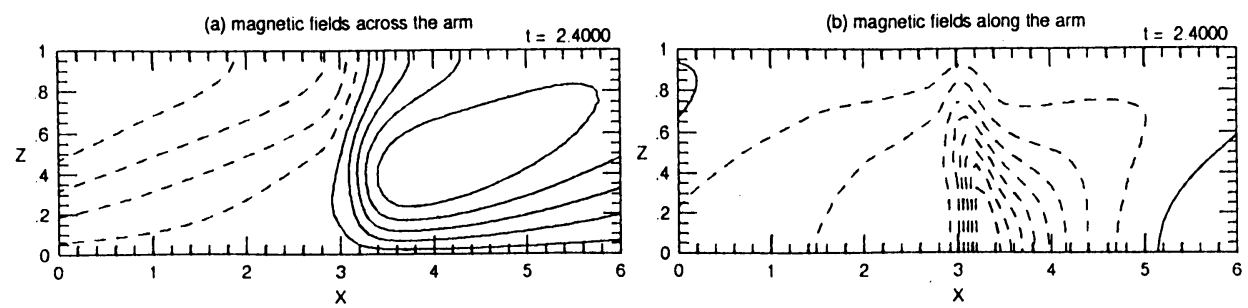

Fig. 1. Meridional pattern of magnetic fields in the model 1 (linear stage)
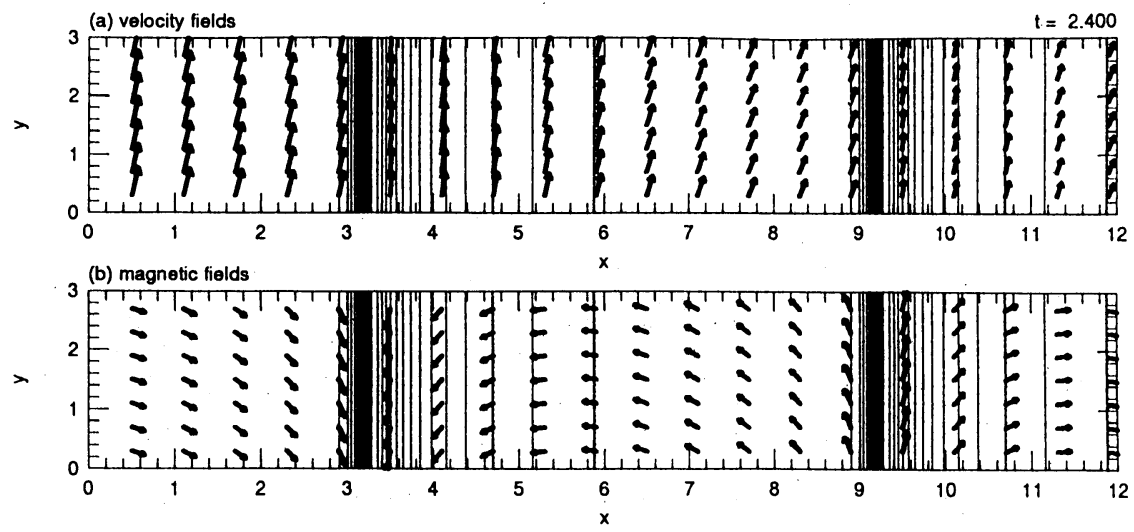

Fig. 2. Face-on-views of velocity (a) and magnetic (b) vectors in the model 1

In the model 1 , the field configuration relative to the arm does not change due to the corotation in the azimuth. Fig.1 shows the meridional pattern of magnetic fields. In this case, the magnetic fields turn to become dynamically important in the later stage and then the further growth of the fields cannot be attained. We found that the field pattern in the steady state are essentially the same as those obtained in the kinematic stage of the swing excitation. Hence, the final field structure is essentially determined in the resonantly interacted state of the magnetic fields with spiral arms.

Fig.2 is the face-on view of the velocity (a) and magnetic vectors (b) in the model 1. The contours denote gas density. It is remarkable that the magnetic vectors cross the velocities in the inter-arm regions with relatively weak strength. When we map these patterns to the usual cylindrical frame, the magnetic fields should be aligned with spiral arms, as suggested by observational results. In other models, the magnetic fields are parallel to velocity fields, not parallel to spiral arms. Therefore, it is concluded that only the model 1 is consistent with observations.

The author acknowledges the Alexander von Humboldt Foundation for support.

\section{References}

Tubbs, A.D. 1980, A p.J., 239, 882. 